\title{
Validación de un procedimiento antropométrico, para estimación de peso seco en pacientes de 6 a 13 años con afecciones renales (1)
}

\author{
Cecilia Carolina Hermosilla Samayoa*
}

\section{Resumen}

La enfermedad renal crónica en estadío terminal (ERC-V), según datos a nivel internacional, afecta entre 1 a 3 niños por cada millón de la población total. La medición del peso corporal de la población infantil, es importante para determinar sus requerimientos nutricionales; sin embargo, los pacientes renales tienden a presentar balance positivo de líquidos y edema, por lo que la determinación del peso corporal real es imprecisa. Este estudio busca validar un procedimiento antropométrico para estimación de peso seco en pacientes guatemaltecos de 6 a 13 años, con afecciones renales crónicas que asisten a la Fundación para el Niño con Enfermedad Renal de Guatemala. El estudio comprendió dos fases, en la primera se aplicaron las ecuaciones antropométricas de Ramírez, Laboratorios Ross y Chumlea-Guo-Roche Steinbaugh. Dichas propuestas, originalmente disponibles a nivel nacional para estimar el peso en adultos. Al aplicarse a 203 niños, aparentemente sanos, para determinar correlación y desviación entre peso estimado y peso real; se requirieron ajustes pertinentes para aumentar su precisión. Durante la segunda fase, para validación de las ecuaciones ajustadas en 54 niños con ERC-V y síndrome nefrótico, se realizó un análisis mediante un modelo de regresión múltiple y se formularon ecuaciones, a partir de talla, circunferencia media de brazo, perímetro mínimo de cuello y perímetro de pantorrilla; que son las medidas que mejor explican el peso de estos niños. Se obtuvieron cuatro ecuaciones, cuya validación resulta en una desviación promedio de $1.97 \mathrm{~kg}$, al aplicarse en niños con ERC-V o síndrome nefrótico. El procedimiento validado para estimación de peso seco, consta de la toma de medidas, mediante la técnica aplicada en este estudio, y sustitución de valores en la ecuación correspondiente para niños de 6 a 9 años.

Palabras clave: validación, procedimiento, antropometría, estimación, peso seco, niños, pediatría, afecciones renales.

* El presente texto de la autora, revisado por Daniel Frade y Judith López de la Facultad de Ciencias de la Salud de la Universidad Rafael Landívar, es la exposición resumida de la tesis para optar al grado de licenciada en Nutrición. 


\section{Abstract}

According to international data, chronic kidney disease in the terminal stage (CKD V) is approximately 1-3 children per million of the total population. The body weight of the infant population is important to determine nutritional requirements and renal patients tend to have a positive fluid balance and edema, so the determination of real body weight is imprecise. This study seeks to validate an anthropometric procedure for the estimation of dry weight in Guatemalan patients aged 6 to 13 years with chronic renal conditions attending the Fundación para el Niño con Enfermedad Renal [Fundation for Childrens with Kidney Disease] in Guatemala. The study include two phases, in the first the anthropometric equations of Ramírez, Ross Laboratories and Chumlea-Guo-Roche Steinbaugh, originally proposed to estimate weight in adults and available nationally, were applied to 203 apparently healthy children, to determine the correlation and the deviation between estimated weight and real weight, relevant adjustments were determined to increase their accuracy. The second phase for validation of adjusted equations in 54 children with CKD V and nephrotic syndrome. An analysis was made using a multiple regression model and equations were formulated, starting with size, average arm circumference, minimum neck circumference and calf perimeter, which are the measures that best explain the weight of these children. Four equations were obtained, whose validation results in an average deviation of $1.97 \mathrm{~kg}$, when applied in children with CKD V or nephrotic syndrome. The validated procedure for estimating dry weight consists of taking measurements using the technique applied in this study and replacing values in the corresponding equation, for children from 6 to 9 years old.

Keywords: validation, procedure, anthropometry, estimation, dry weight, children, pediatrics, renal affections. 


\section{Introducción}

Las afecciones renales durante la infancia, tienden a diagnosticarse por sintomatología, que puede llegar a confundirse con anemia microcítica-hipocrómica, cefalea o por la presencia de edema. Ello ocurre, por lo regular, cuando la enfermedad se encuentra en estadíos avanzados. La uremia en estos niños tiende a provocar anorexia secundaria y malabsorción (1, p. 341); además, la dieta suele ser muy restrictiva desde el momento del diagnóstico. Por ello y otra serie de factores, dicha población presenta en su mayoría, desnutrición proteico-energética (2, pp. 91-94).

Es importante aclarar que estos niños necesitan un plan de recuperación nutricional para mejorar su calidad de vida, la oportunidad que tengan de optar por un trasplante de riñón y la disminución en la recurrencia de infecciones. Este plan debe iniciarse, tan pronto como sea posible, por lo que se contempla el peso seco del niño que permite determinar los requerimientos nutricionales, la ganancia de peso progresiva y la mejora de la relación peso/talla.
El peso seco se define como el menor peso corporal que tolera una persona, previo a presentar complicaciones por desbalances hidroelectrolíticos (3, p. 392).

Los pacientes renales, sobre todo aquéllos que padecen síndrome nefrótico, tienden a presentar un balance positivo de líquidos y edema (4, p. 1152; 5, p. 1246). La valoración del peso corporal libre de edema, en pacientes renales, es difícil e imprecisa. Pueden utilizarse ecuaciones derivadas del análisis de composición corporal u otros métodos, de modo que se determine el peso con el menor contenido de agua posible (1, p. 19). Se sabe de métodos desarrollados para estimar peso, mediante antropometría en la población adulta. A nivel nacional, se dispone de las ecuaciones de Ramírez (RA), Laboratorios Ross (LR) y Chumlea-Guo-Roche Steinbaugh (CGRS) (1, p. 1; 6, pp. 7-8; 7, pp. 564-568) y de procedimientos no antropométricos, validados en poblaciones pediátricas de otros países con afecciones renales, como la determinación del grosor de la vena cava mediante ecografía (8, pp. 1203-1206); aunque ha sido cuestionado en otros estudios, dado que mide el volumen sanguíneo, pero no necesariamente el peso seco (9, pp. 1066-1072).
Se sabe también de procedimientos antropométricos, validados en poblaciones pediátricas de otras nacionalidades, para determinar peso de forma rápida en servicios de emergencias, pero no tienen especificidad para niños con afecciones renales, ni se usan a nivel nacional (10, pp. 160-165). La población atendida por la Fundación para el Niño con Enfermedad Renal (Fundanier) se encuentra, comprendida entre los 6 a 13 años de edad, por lo que se procedió a la revisión de ecuaciones disponibles a nivel nacional, para estimar peso y se validó un procedimiento antropométrico, para así mejorar la atención a nivel clínico.

\section{Materiales y métodos}

Fase 1: Muestra

Se evaluó a 203 niños aparentemente sanos, entre los 6 a 13 años, inscritos en un centro educativo público del departamento de Guatemala, debido a que su situación socioeconómica y cultural era similar a la población objetivo. Estos niños debían ser capaces de permanecer en bipedestación, durante al menos 10 minutos, sin ayuda u apoyo, para poder evaluarse antropométricamente de forma adecuada; además no padecer trastornos neurológicos, ni enfermedades que implicaran presencia de tumores o edema.

Fase 1: Medición

A cada niño participante se le asignó un código numérico. Se recolectó de cada uno, los valores de peso, talla y las siete medidas antropométricas que requieren las ecuaciones de RA, 
LR y CGRS (ver tabla 1). El equipo antropométrico utilizado incluyó una balanza electrónica profesional marca Tanita, un tallímetro marca SECA, una cinta métrica flexible graduada en centímetros; así como un cáliper profesional. Los datos se anotaron manualmente, en hojas individuales. Se realizó la toma de medidas a cada paciente, duró en promedio cinco minutos, previa estandarización. Las técnicas antropométricas utilizadas se pueden consultar en el Anthropometric Standarization Reference Manual (11, p. 177) y en Guzmán et al (12, pp. 358-363). A partir de estos datos, se identificaron las medidas que mejor explicaban el peso de la muestra y se adecuaron las ecuaciones para la población objetivo.

Tabla 1. Ecuaciones de referencia (RA, LR y CGRS)

\begin{tabular}{|c|c|c|c|c|c|}
\hline \multirow{2}{*}{ Autor } & \multicolumn{5}{|c|}{ Ecuación } \\
\hline & \multicolumn{3}{|c|}{ Hombres } & \multicolumn{2}{|r|}{ Mujeres } \\
\hline \multirow{4}{*}{ RA } & \multirow{4}{*}{ Peso $=$} & & 0.29 * EB & \multirow{4}{*}{ Peso $=$} & $0.25 * \mathrm{~EB}$ \\
\hline & & + & 2.12 * $P A B$ & & +2.12 * $\mathrm{PAB}$ \\
\hline & & + & 1.79 * PMC & & $+1.79 * \mathrm{PMC}$ \\
\hline & & & 108.69 & & - 57.78 \\
\hline \multirow{3}{*}{ LR } & \multirow{3}{*}{ Peso $=$} & & 1.19 * $A R$ & \multirow{3}{*}{ Peso $=$} & $1.01 * A R$ \\
\hline & & + & $3.21 \mathrm{CMB}$ & & $+2.81 \mathrm{CMB}$ \\
\hline & & - & 86.82 & & -66.04 \\
\hline \multirow{5}{*}{ CGRS } & \multirow{5}{*}{ Peso = } & & 0.98 * PPA & \multirow{5}{*}{ Peso $=$} & 1.27 * PPA \\
\hline & & + & 1.16 * $A R$ & & $+\quad 0.87 * A R$ \\
\hline & & + & $1.73 * \mathrm{CMB}$ & & 0.98 * $\mathrm{CMB}$ \\
\hline & & + & 0.37 * PS & & $+\quad 0.4$ *PS \\
\hline & & - & 81.69 & & $-\quad 62.35$ \\
\hline
\end{tabular}

$\mathrm{EB}=$ Extensión de brazada $(\mathrm{cm})$

$\mathrm{PAB}=$ Perímetro de antebrazo $(\mathrm{cm})$

$\mathrm{PMC}=$ Perímetro mínimo de cuello $(\mathrm{cm})$

$\mathrm{CMB}=$ Circunferencia media del brazo $(\mathrm{cm})$

$\mathrm{AR}=$ Altura de la rodilla $(\mathrm{cm})$

PPA $=$ Perímetro de pantorrilla $(\mathrm{cm})$

Nota: la tabla 1, presenta las ecuaciones de referencia, de las fórmulas utilizadas para el estudio.

Fase 2: Muestra para validación

La muestra fue de 54 niños atendidos en Fundanier, que padecen ERC-V o síndrome nefrótico, cuyos diagnósticos no coexistían con trastornos neurológicos $u$ otras patologías que implicaran presencia de tumores. Además, debían ser capaces de permanecer en bipedestación durante diez minutos, sin ayuda u apoyo.

Fase 2: Medición para validación

A cada niño participante se le asignó un código numérico.

Se recolectó de cada uno, los valores de talla, CMB, PMC y PPA, además del peso seco obtenido por el método que normalmente utiliza Fundanier. El equipo antropométrico utilizado, incluyó una balanza electrónica profesional marca Health-o-meter, un tallímetro marca SECA y una cinta métrica flexible graduada en centímetros. Los datos se anotaron manualmente. 
real y el peso estimado por cada ecuación durante la fase 1 . Se identificaron ajustes pertinentes, para adaptar estas ecuaciones mediante un modelo analítico de regresión lineal múltiple.

Durante la fase 2, se determinaron valores de estadística descriptiva (Error estándar, coeficiente de correlación, $R^{\wedge} 2$ ), así como el grado de desviación entre el peso real y el peso estimado. Todo el análisis estadístico de ambas fases se realizó con un nivel de confianza del $95 \%$ $(p<0.05)$, mediante el empleo del programa Excel de Microsoft Office 2010 de la Corporación Microsoft.

\section{Resultados}

Se obtuvieron datos de la primera fase, con un total de 115 niños y 88 niñas. Se determinó el intervalo de rango de desviación y la desviación promedio entre peso.

\section{Debido a que estas mostraban} intervalos de rangos de desviación muy amplios, se aplicó regresión lineal múltiple a todas las medidas obtenidas. El estudio prescindió de la EB, que fue sustituida por la talla. Gracias a esto se determinó que las mejores medidas para explicar el peso $(p<0.05)$, en todos los casos, son: talla, CMB, PMC y PPA. Del total de 203 participantes, se excluyeron los datos de 15, porque presentaban inadecuado estado nutricional por exceso. Se verificó que el error permitido no superara el $5 \%$, luego de la depuración, dada la poca similitud que presentan en su mayoría con la población objetivo, y el estado nutricional en riesgo o inadecuado por déficit.

Puesto que ninguna de las ecuaciones de referencia utiliza esta combinación de medidas, se efectúo nuevamente la regresión lineal múltiple para la muestra. Los mejores resultados se obtuvieron al dividirla, según sexo y dos grupos de edades: de 6-9 años y de 10-13 años (ver tabla 2).

De los niños participantes durante la fase 2, uno padecía un problema hormonal, por lo que su crecimiento era inadecuado. Se observó en sus resultados que las ecuaciones no predecían adecuadamente su peso en específico, debido a una gran diferencia de estatura con la muestra de referencia. Por ello no se utilizaron sus datos.

Tabla 2. Resumen de estadísticas de ecuaciones desarrolladas

$\begin{array}{lcccr}\text { Dato } & \text { Grupo de } 6 \text { a } 9 \text { años } & \text { Grupo de } 10 \text { a } 13 \text { años } \\ & \text { niños } & \text { niñas } & \text { niños } & \text { niñas } \\ \text { Número de observaciones } & & & & 39 \\ \text { Coeficiente de correlación múltiple } & 60 & 40 & 0.9848 & 0.9936 \\ \text { Coeficiente de determinación R ^2 } & 0.9872 & 0.9876 & 0.9698 & 0.9873 \\ \mathrm{R} \wedge 2 \text { ajustado } & 0.9747 & 0.9754 & 0.9671 & 0.9858 \\ \text { Error estándar } & 0.9728 & 0.9726 & 1.7599 & 1.1577\end{array}$

Nota: en la tabla se resume el análisis de regresión lineal múltiple, dividida en sexo y dos grupos de edades. 
Los valores estadísticos mejoraron al depurar la muestra, puesto que tres participantes de la fase 2 presentaban valores de CMB por debajo de la muestra de referencia $(>-1.5 \mathrm{~cm})$, (ver tabla 3$)$.

Tabla 3. Resumen de datos estadísticos sobre la validación de ecuaciones en muestra depurada

$\begin{array}{lc}\text { Dato } & \text { valor } \\ \text { Observaciones } & 50 \\ \mathrm{R} \text { (entre peso real y peso permitido) } & 0.9748 \\ \mathrm{R} \wedge 2 & 0.9502 \\ \text { Error estándar }(\mathrm{kg}) & 1.04 \\ \text { Intervalo de rango de desviación }(\mathrm{kg}) & -3.8 \text { a } 4.7 \\ \text { Desviación promedio }(\mathrm{kg}) & 1.70\end{array}$

$\mathrm{R} \quad=$ Coeficiente de relación

$\mathrm{R} \wedge 2$ = Coeficiente de determinación

Nota: en la tabla 3, se resumen los resultados al depurar a los participantes con valores de $\mathrm{CMB}$ por debajo de la muestra de referencia.

\section{Discusión}

Los datos obtenidos en la fase 1 de la investigación, demuestran que las tres ecuaciones de referencia, sin modificaciones, no predicen de forma exacta el peso corporal de niños de 6 a 13 años. Esto se debe a claras diferencias de composición corporal, además en el caso de las poblaciones de referencia en las ecuaciones LR y CGRS, pertenecen a países desarrollados, con hábitos de alimentación distintos a los de Guatemala, cuyos resultados de estudios no son multicéntricos, para sustentar su validez internacional. Por otro lado, la ecuación de Ramírez fue diseñada para pacientes inmovilizados y los coeficientes resultaron de técnicas de medición para pacientes encamados; por lo que resulta inapropiado usarlas con pacientes en bipedestación.

De las medidas recolectadas se decidió prescindir de EB, pues en los niños de 6-9 años, los huesos se encuentran aún en formación. Eran incapaces de extender su brazo de forma recta presentaban una curva a nivel del codo, por la presencia de cartílago que dificultaba la medición. En general, los niños de 10-13 años tendían a presentar, fisiológicamente, una medida mucho mayor de EB que de talla, porque aún no habían alcanzado el punto máximo de crecimiento de la adolescencia; aunque sus brazos empezaban a alongarse. Además, esta medida fue incluida en la ecuación de RA para pacientes inmovilizados, es la forma más sencilla de determinar la talla. Puesto que a la población objetivo pudo determinársele la talla, se sustituyó para disminuir error. 
En general, el valor de PMC y PPA tendían a parecerse en ambas muestras. Si el PPA era un valor más alejado al PMC, se trataba de un niño clínicamente más delgado y con un $\mathrm{CMB}$ menor a otro participante con valores de PMC y PPA más cercanos, clínicamente más robusto y con un CMB mayor.

Las ecuaciones se aplicaron, con las mismas medidas, pero según sexo y edad, puesto que en la pubertad empiezan a diferenciarse los cuerpos de niños y niñas. También se trabajó con un rango amplio de edad, que permite, a su vez, rangos mayores en el valor de cada medida de las observaciones; finalmente, el error estándar aumentaba y la precisión disminuía. Se dividió la muestra en dos grupos de edades, el primero de 6-9 años, porque son niños que, en general, no han iniciado la pubertad; aunque empiezan a distinguirse a nivel de proporciones corporales. El segundo grupo de 10-13 años, son niños que iniciaron la pubertad $y$, en algunos casos, su adolescencia y desarrollo fisiológico. En los niños de 10-13 años ya marcan, evidentemente, distintos ritmos de desarrollo. Algunos aún no lo inician, otros lo inician tempranamente y esto da lugar a que, por ejemplo, niñas con las mismas medidas, arrojen pesos estimados iguales. Una de ellas ha desarrollado busto, aumentó su peso y se desvió el valor de peso estimado.
Las niñas presentaron, en la estimación de su peso, valores de desviación estándar mayores que los de niños de su misma edad. Esto se puede deber a que en ellas, son más evidentes las diferencias corporales individuales, dada la mayor reserva grasa que fluctúa, según su alimentación, genética, actividad física, etc.

Durante la fase 2 de la investigación se pudo observar que algunos valores de desviación del dato estimado y del dato real, eran mayores para niños, su CMB era más bajo que los obtenidos para su edad y sexo, en la muestra de referencia para la fase 1. Las ecuaciones obtenidas son válidas, siempre que se utilicen en poblaciones similares a las usadas para desarrollarlas. En general, se buscó similitud entre población de referencia con la población objetivo; proveniente de familias con un ingreso económico similar, con el mismo rango de edades y que presentan estados nutricionales parecidos (por esto se excluyó a niños con sobrepeso y obesidad). Es posible encontrar casos con valores de medida fuera del rango de referencia y muestren una desviación mayor que la promedio.

Se sugiere que el procedimiento resultante se complete de forma rutinaria en Fundanier, u otros servicios de salud que evalúen a esta población como complemento a la evaluación nutricional. Se debe de tener en cuenta, que la utilización de equipo profesional disminuye el margen de error en la 
medición y que debe monitorizarse la aplicación adecuada del procedimiento, así como la calibración del equipo. El procedimiento antropométrico para estimación de peso seco, consta de dos partes: toma de medidas mediante la técnica aplicada en este estudio (ver apéndice 1) y sustitución de valores de cada medida, en la ecuación correspondiente, según edad y sexo, para cada caso.

\section{Conclusiones}

La precisión de las ecuaciones evaluadas en la fase 1, no es apropiada para usarse como tales en niños de 6 a 13 años; según la desviación promedio respecto del dato real que es de $4.63 \mathrm{~kg}$.

Las ecuaciones validadas para estimación de peso seco $(\mathrm{kg})$ son las siguientes:

1. Para niños de 6 a 9 años: peso $=(0.245 *$ talla $)+(0.766 * C M B)$ $+(0.546$ * $P M C)+(0.456$ *PPA $)-46.25$

2. Para niños de 10 a 13 años: peso $=(0.317$ * talla $)+(0.920$ * $\mathrm{CMB})+(0.488$ * $\mathrm{PMC})+(0.957$ * PPA $)-70.74$

3. Para niñas de 6 a 9 años: peso $=(0.248 *$ talla $)+(0.696 * \mathrm{CMB})$ $+(0.795 * \mathrm{PMC})+(0.492 * \mathrm{PPA})-52.84$

4. Para niñas de 10 a 13 años: peso $=(0.272$ * talla $)+(1.057$ * $\mathrm{CMB})+(0.672$ * $\mathrm{PMC})+(1.001$ * PPA $)-73.6$

Las ecuaciones son válidas, siempre que los niños se encuentren dentro de los rangos de medidas de la población sana de referencia, de lo contrario los resultados se alejan del valor real.

Agradecimientos

A la Lcda. Victoria Rodríguez y el Ing. Freddy Girón, por su asesoría, así como a todos los profesionales involucrados; a Fundanier y a la Universidad Rafael Landívar. 


\section{Referencias}

1. de la Mano Hernández A, Moráis López A. Nutrición en situaciones especiales: Enfermedad renal crónica y enfermedad oncológica. En: Sociedad Española de Pediatría (AEP), ed. Protocolos diagnóstico-terapéuticos de Gastroenterología, Hepatología y Nutrición Pediátrica. 2da ed. Madrid: AEP. 2010; 341-346.

2. Fischbach M., Dheu C., Seuge L., \& Orfanos L. Hemodialysis and Nutritional Status in Children: Malnutrition and Cachexia. Journal of Renal Nutrition. 2009; 19(1): 91-94. DOI: 10.1053/j.jrn.2008.10.008

3. Jaeger J. \& Mehta R. Assessment of Dry Weight in Hemodialysis: An Overview. J Am Soc Nephrol. 1999; 10: 392-403

4. Guyton A. y Hall J. Tratado de fisiología médica. 11va Ed. Madrid: Elsevier. 2006; $1152 \mathrm{p}$.

5. Nelson W., Vaughan V. \&, McKay J. Tratado de pediatría. 7ma Ed. México: Salvat. 1980; $1246 \mathrm{p}$.

6. Rabat J. y Rebollo I. Estimación de peso y talla [internet]. España. Recuperado a partir de: http://www.sancyd.es/luis/tablas/99032.pdf 
7. Chumlea W. C., Guo S., Roche A. \& Steinbaugh L. Prediction of body weight for the nonambulatory elderly from anthropometry. J Am Diet Assoc. 1988; 88(5): 564-8;

8. Krause I., Birk E., Davidovits M., Cleper R., Blieden L., Pinhas L., Gamzo Z. \& Eisenstein B. Inferior vena cava diameter: a useful method for estimation of fluid status in children on haemodialysis. Nephrol Dial Transplant. 2001; 16: 1203-1206; .

9. Agarwal R., Bouldin M., Light R. \& Garg A. Inferior vena cava diameter and left atrial diameter measure volume but not dry weight. Clin J Am Soc Nephrol. 2011; (6) 1066-1072.

10. Black K., Barnett P., Wolfe R. \& Young S. Are methods used to estimate weight in children accurate? Emergency Medicine. 2002; 14: 160-165;

11. Lohman T., Roche A. \& Martorell R. Anthropometric Standarization Reference Manual. Illinois: Human Kinetics Books. 1988; 177 p.

12. Guzmán C., Reinoza G. y Hernández R. A. Estimación de la estatura a partir de la longitud de la pierna medida con cinta métrica. Nutr. Hosp. Sept. / oct. 2005; 20(5): 358-363. 


\section{Apéndice 1 \\ Técnicas para la toma de medidas**}

\section{Medición de la talla}

- El niño debe medirse sin zapatos, calcetines / calcetas, adornos para el cabello, colas de caballo, u otros objetos que obstaculicen la toma de la medida (figura 1).

- Debe pararse de espaldas a la escala del tallímetro, en posición erguida, con las rodillas estiradas, las piernas rectas; y los pies formando un ángulo cómodo para el paciente, no paralelos.

- Se debe respetar el plano de Frankfort (figura 2). Los brazos deben descansar a los costados.

- La medida se toma presionando el cabello, en el milímetro más cercano (figura 3).
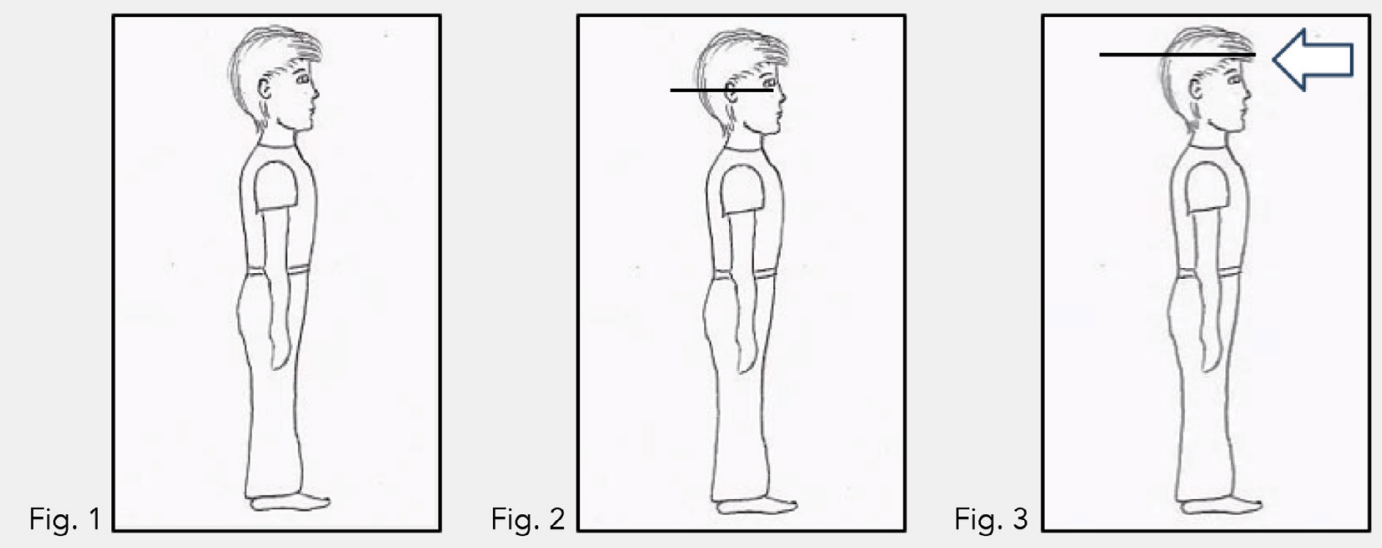

Medición de la circunferencia media del brazo (CMB)

- El niño debe descubrir su brazo no dominante, por completo.

- Se solicita colocar el brazo de modo que el codo forme un ángulo de 90 (figura 1).

- Se localiza el acromión y se lleva la cinta métrica, desde este punto, hacia el olécranon.

- Se localiza el punto medio entre el acromión y el olécranon, se marca el punto (figura 2).

- Se solicita relajar el brazo y se rodea el mismo, debajo de la marca, con la cinta métrica (figura 3).

- La medida se toma en el milímetro más cercano, sin compresionar tejidos.

Fig. 1

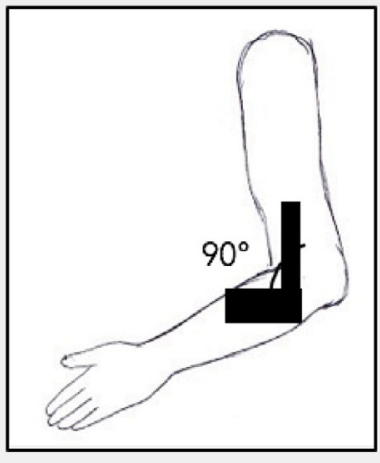

Fig. 2

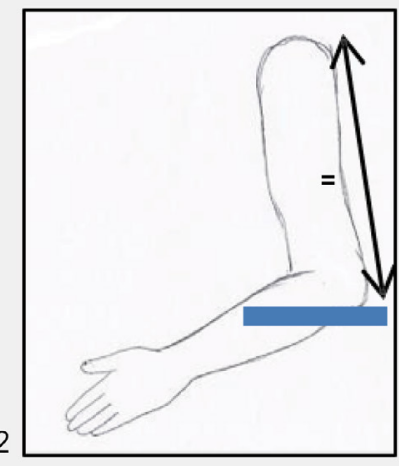

Fig. 3

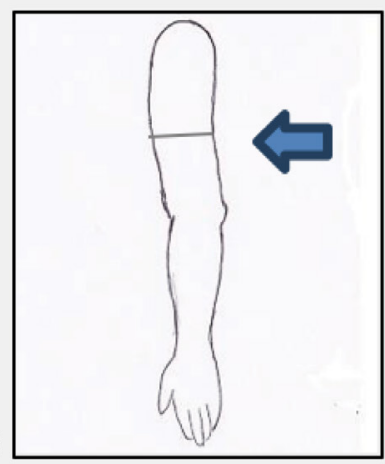

\footnotetext{
** Timothy G. Lohmann, Alex F. Roche, and Reynaldo Martorell. Anthropometric Standarization Reference Manual.

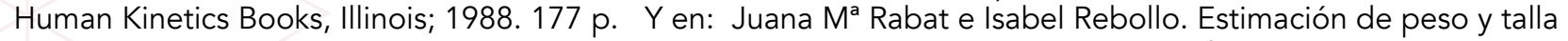
[internet]. España. Recuperado a partir de: http://www.sancyd.es/luis/tablas/99032.pdf
} 
Medida del perímetro mínimo de cuello (PMC)

- El niño no debe tener accesorios alrededor del cuello (figura 1).

- Debe posicionarse erguido y respetar el plano horizontal de Frankfort (figura 2).

- La cinta se coloca debajo de la prominencia laringeal (si existe) y se forma un ángulo de $90^{\circ}$, en relación con el axis largo.

- La medida se toma en el milímetro más cercano, sin compresionar tejidos (figura 3).

Fig. 1

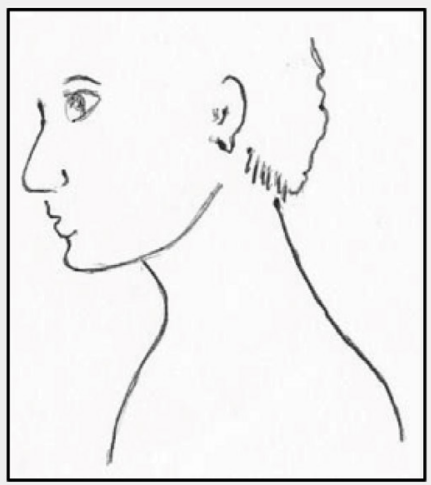

Fig. 2
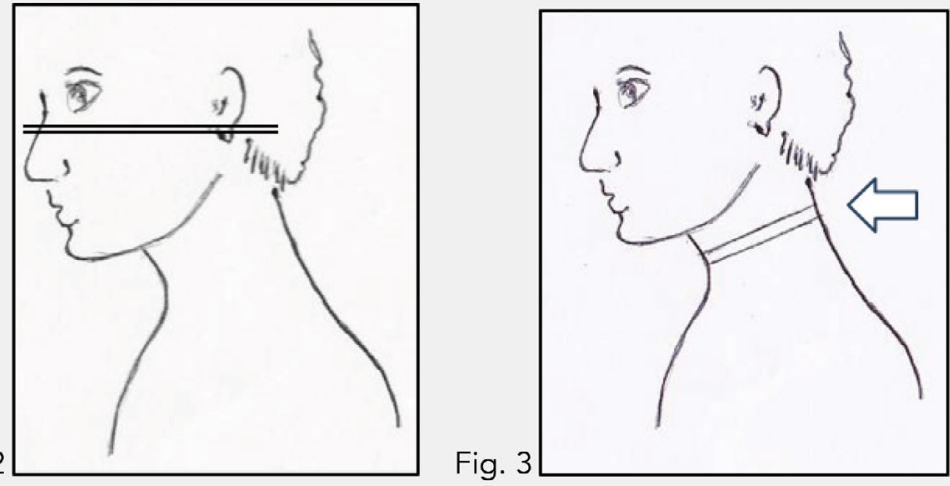

Medida del perímetro de la pantorrilla (PPA)

- El niño debe colocarse en posición supina y descubrir su pierna del lado no dominante, hasta la rodilla.

- Debe recoger la pierna, hasta que su rodilla forme un ángulo de $90^{\circ}$ (figura 1).

- La cinta métrica debe colocarse alrededor de la pantorrilla; y llevarse hacia arriba y hacia abajo, hasta localizar el punto más prominente, en el que se toma la medida (figura 2).

- La medida se toma en el milímetro más cercano, sin compresionar tejidos.

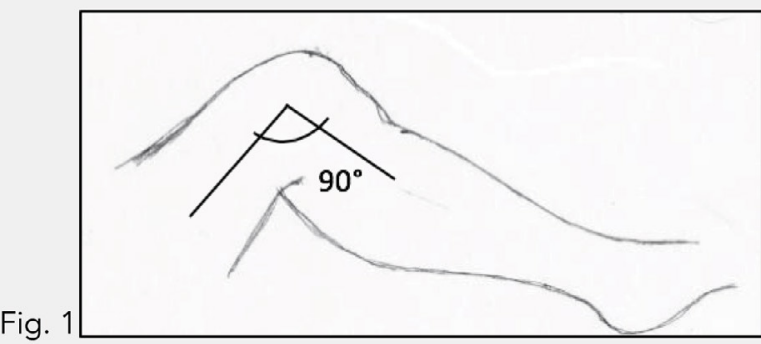

Fig. 2

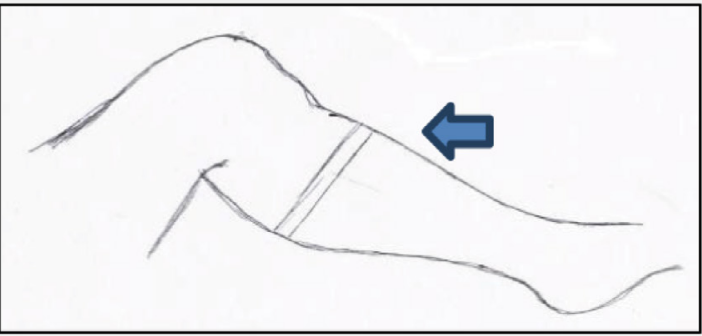

